ISSN 1855-3966 (printed edn.), ISSN 1855-3974 (electronic edn.)

ARS MATHEMATICA CONTEMPORANEA 16 (2019) 609-623

https://doi.org/10.26493/1855-3974.1681.4ec

(Also available at http://amc-journal.eu)

\title{
Embedding of orthogonal Buekenhout-Metz unitals in the Desarguesian plane of order $q^{2}$
}

\author{
Gábor Korchmáros, Alessandro Siciliano \\ Dipartimento di Matematica, Informatica ed Economia, \\ Università degli Studi della Basilicata, \\ Viale dell'Ateneo Lucano 10 - 85100 Potenza (Italy)
}

Received 19 April 2018, accepted 18 February 2019, published online 5 June 2019

\begin{abstract}
A unital, that is a $2-\left(q^{3}+1, q+1,1\right)$ block-design, is embedded in a projective plane $\pi$ of order $q^{2}$ if its points are points of $\pi$ and its blocks are subsets of lines of $\pi$, the point-block incidences being the same as in $\pi$. Regarding unitals $\mathcal{U}$ which are isomorphic, as a block-design, to the classical unital, T. Szónyi and the authors recently proved that the natural embedding is the unique embedding of $\mathcal{U}$ into the Desarguesian plane of order $q^{2}$. In this paper we extend this uniqueness result to all unitals which are isomorphic, as block-designs, to orthogonal Buekenhout-Metz unitals.
\end{abstract}

Keywords: Unital, embedding, finite Desarguesian plane.

Math. Subj. Class.: 51E05, 51E20

\section{Introduction}

A unital is a set of $q^{3}+1$ points equipped with a family of subsets, each of size $q+1$, such that every pair of distinct points are contained in exactly one subset of the family. In Design Theory, such subsets are usually called blocks so that unitals are $2-\left(q^{3}+1, q+1,1\right)$ block-designs. A unital $\mathcal{U}$ is embedded in a projective plane $\pi$ of order $q^{2}$, if its points are points of $\pi$, its blocks are subsets of lines of $\pi$ and the point-block incidences being the same as in $\pi$.

Sufficient conditions for a unital to be embeddable in a projective plane are given in [21]. Computer aided searches suggest that there should be plenty of unitals, especially for small values of $q$, but those embeddable in a projective plane are quite rare, see [3, 6, 27]. Very recently, the GAP package UnitalSz was released [25]. This package contains methods for the embeddings of unitals in the finite projective plane.

E-mail addresses: gabor.korchmaros@unibas.it (Gábor Korchmáros), alessandro.siciliano@unibas.it (Alessandro Siciliano)

(a)(i) This work is licensed under https://creativecommons.org/licenses/by/4.0/ 
In the finite Desarguesian projective plane of order $q^{2}$, a unital arises from a unitary polarity: the points of the unital are the absolute points, and the blocks are the non-absolute lines of the polarity. This unital is called classical unital. The following result comes from [23].

Theorem 1.1. Let $\mathcal{U}$ be a unital embedded in $\mathrm{PG}\left(2, q^{2}\right)$ which is isomorphic, as a blockdesign, to a classical unital. Then $\mathcal{U}$ is the classical unital of $\operatorname{PG}\left(2, q^{2}\right)$.

Buekenhout [11] constructed unitals in any translation planes with dimension at most two over their kernel by using the Andrè/Bruck-Bose representation. Buekenhout's work was completed by Metz [24] who was able to prove by a counting argument that when the plane is Desarguesian then Buekenhout's construction provides not only the classical unital but also non-classical unitals in $\operatorname{PG}\left(2, q^{2}\right)$ for all $q>2$. These unitals are called Buekenhout-Metz unitals, and they are the only known unitals in $\operatorname{PG}\left(2, q^{2}\right)$. With the terminology in [5], an orthogonal Buekenhout-Metz unital is a Buekenhout-Metz unital arising from an elliptic quadric in Buekehout's construction.

In this paper, we prove the following result:

Main Theorem. Let $\mathcal{U}$ be a unital embedded in $\mathrm{PG}\left(2, q^{2}\right)$ which is isomorphic, as blockdesign, to an orthogonal Buekenhout-Metz unital. Then $\mathcal{U}$ is an orthogonal BuekenhoutMetz unital.

Our approach is different from that adopted in [23]. Our idea is to exploit two different models of $\mathrm{PG}\left(2, q^{2}\right)$ in $\mathrm{PG}(5, q)$, one of them is a variant of the so-called $\mathrm{GF}(q)$-linear representation. We start off with a representation of a non-classical Buekenhout-Metz unital given in one of these models of $\operatorname{PG}\left(2, q^{2}\right)$, then we exhibit a linear collineation of $\operatorname{PG}(5, q)$ that takes this representation to a representation of a classical unital in the other model of $\mathrm{PG}\left(2, q^{2}\right)$. At this point to finish the proof we only need some arguments from the proof of Theorem 1.1 together with the characterization of the orthogonal Buekenhout-Metz unitals due to Casse, O'Keefe, Penttila and Quinn [12, 29].

\section{Preliminary results}

The study of unitals in finite projective planes has been greatly aided by the use of the Andrè/Bruck-Bose representation of these planes [1, 9, 10]. Let $\mathrm{PG}(4, q)$ denote the projective 4-dimensional space over the finite field $\mathrm{GF}(q)$, and let $\Sigma$ be some fixed hyperplane of $\operatorname{PG}(4, q)$. Let $\mathcal{N}$ be a line spread of $\Sigma$, that is a collection of $q^{2}+1$ mutually skew lines of $\Sigma$. We consider the following incidence structure: the points are the points of $\operatorname{PG}(4, q)$ not in $\Sigma$, the lines are the planes of $\operatorname{PG}(4, q)$ which meet $\Sigma$ in a line of $\mathcal{N}$ and incidence is defined by inclusion. This incidence structure is an affine translation plane of order $q^{2}$ which is at most two-dimensional over its kernel. It can be completed to a projective plane $\pi(\mathcal{N})$ by the addition of an ideal line $L_{\infty}$ whose points are the elements of the spread $\mathcal{N}$. Conversely, any translation plane of order $q^{2}$ with $\operatorname{GF}(q)$ in its kernel can be modeled this way [9]. Moreover, it is well known that the resulting plane is Desarguesian if and only if $\mathcal{N}$ is a Desarguesian spread [10].

Our first step is to outline the usual representation of $\operatorname{PG}\left(2, q^{2}\right)$ in $\operatorname{PG}(5, q)$ due to Segre [30] and Bose [7]. While such representation is usually thought of in a projective setting, algebraic dimensions are more amenable to an introductory discussion of it, so we will mainly take a vector space approach along all this section. 
Look at $\operatorname{GF}\left(q^{2}\right)$ as the two-dimensional vector space over $\operatorname{GF}(q)$ with basis $\{1, \epsilon\}$, so that every $x \in \operatorname{GF}\left(q^{2}\right)$ is uniquely written as $x=x_{0}+x_{1} \epsilon$, for $x_{0}, x_{1} \in \operatorname{GF}(q)$. Then the vectors $(x, y, z)$ of $V\left(3, q^{2}\right)$ are viewed as the vectors $\left(x_{1}, x_{2}, y_{1}, y_{2}, z_{1}, z_{2}\right)$ of $V(6, q)$ where

$$
\begin{aligned}
& x=x_{0}+x_{1} \epsilon, \\
& y=y_{0}+\epsilon y_{1} \text { and } \\
& z=z_{0}+\epsilon z_{1} .
\end{aligned}
$$

Therefore the points of $\mathrm{PG}\left(2, q^{2}\right)$ are two-dimensional subspaces in $V(6, q)$, and hence lines of $\mathrm{PG}(5, q)$, the five-dimensional projective space arising from $V(6, q)$. Such lines are the members of a Desarguesian line-spread $\mathcal{S}$ of $\mathrm{PG}(5, q)$ which gives rise to a pointline incidence structure $\Pi(\mathcal{S})$ where points are the elements of $\mathcal{S}$, and lines are the threedimensional subspaces of $\operatorname{PG}(5, q)$ spanned by two elements of $\mathcal{S}$, incidence being inclusion. Obviously, $\Pi(\mathcal{S}) \simeq \operatorname{PG}\left(2, q^{2}\right)$, and $\Pi(\mathcal{S})$ is the $\mathrm{GF}(q)$-linear representation of $\operatorname{PG}\left(2, q^{2}\right)$ in $\operatorname{PG}(5, q)$. Since $\operatorname{PG}(5, q)$ is naturally embedded in $\operatorname{PG}\left(5, q^{2}\right)$, we also have an embedding of $\operatorname{PG}\left(2, q^{2}\right)$ in $\mathrm{PG}\left(5, q^{2}\right)$ via $\Pi(\mathcal{S})$.

Actually, we will use a different embedding of $\operatorname{PG}\left(2, q^{2}\right)$ in $\operatorname{PG}\left(5, q^{2}\right)$ which is more suitable for computation.

In $V\left(6, q^{2}\right)$, let $\widehat{V}$ be the set of all vectors $\left(x, x^{q}, y, y^{q}, z, z^{q}\right)$ with $x, y, z \in \operatorname{GF}\left(q^{2}\right)$. With the usual sum and multiplication by scalars from $\operatorname{GF}(q), \widehat{V}$ is a six-dimensional vector space over $\operatorname{GF}(q)$. On the other hand, $V(6, q)$ is naturally embedded in $V\left(6, q^{2}\right)$. Therefore, the question arises whether there exists an invertible endomorphism of $V\left(6, q^{2}\right)$ that takes $\widehat{V}$ to $V(6, q)$. The affirmative answer is given by the following proposition.

Proposition 2.1. $\widehat{V}$ is linearly equivalent to $V(6, q)$ in $V\left(6, q^{2}\right)$.

Proof. Write $V(6, q)$ as the direct sum $W^{(1)} \oplus W^{(2)} \oplus W^{(3)}$, with

$$
\begin{aligned}
& W^{(1)}=\{(a, b, 0,0,0,0): a, b \in \mathrm{GF}(q)\} \\
& W^{(2)}=\{(0,0, a, b, 0,0): a, b \in \operatorname{GF}(q)\} \\
& W^{(3)}=\{(0,0,0,0, a, b): a, b \in \operatorname{GF}(q)\} .
\end{aligned}
$$

Clearly, each $W^{(i)}$ is isomorphic to $V(2, q)=\{(a, b): a, b \in \mathrm{GF}(q)\}$. Take a basis $\left\{u_{1}, u_{2}\right\}$ of $V(2, q)$ together with a Singer cycle $\sigma$ of $V(2, q)$. Since $\sigma$ has two distinct eigenvalues, both in $\operatorname{GF}\left(q^{2}\right) \backslash \mathrm{GF}(q)$, we find two linearly independent eigenvectors $v_{1}, v_{2}$ that form a basis for $V\left(2, q^{2}\right)$. Such a basis $\left\{v_{1}, v_{2}\right\}$ is called a Singer basis with respect to $V(2, q)$ [15]. In this context, $V(2, q)=\left\{x v_{1}+x^{q} v_{2}: x \in \mathrm{GF}\left(q^{2}\right)\right\}$ [14].

Applying this argument to $W^{(i)}$ with $i=1,2,3$, gives a Singer basis $\left\{v_{1}^{(i)}, v_{2}^{(i)}\right\}$ of $W^{(i)}$ such that $W^{(i)}=\left\{x v_{1}^{(i)}+x^{q} v_{2}^{(i)}: x \in \mathrm{GF}\left(q^{2}\right)\right\}$. In this basis we have

$$
V(6, q)=\left\{x v_{1}^{(1)}+x^{q} v_{2}^{(1)}+y v_{1}^{(2)}+y^{q} v_{2}^{(2)}+z v_{1}^{(3)}+z^{q} v_{2}^{(3)}: x, y, z \in \operatorname{GF}\left(q^{2}\right)\right\} .
$$

Now, the result follows from the fact that the change from any basis of $V\left(6, q^{2}\right)$ to the basis $\left\{v_{1}^{(i)}, v_{2}^{(i)}: i=1,2,3\right\}$ is carried out by an invertible endomorphism over $\operatorname{GF}\left(q^{2}\right)$.

We call the vector space $\widehat{V}$ the cyclic representation of $V(6, q)$ over $\operatorname{GF}\left(q^{2}\right)$. 
To state Proposition 2.1 in terms of projective geometry, let $\mathrm{PG}(5, q)$ denote the projective space arising from $V(6, q)$. Also, let $\mathrm{PG}(\widehat{V})=\left\{\langle v\rangle_{q}: v \in \widehat{V}\right\}$ be the five-dimensional projective space whose points are the one-dimensional $\mathrm{GF}(q)$-subspaces spanned by vectors in $\widehat{V}$.

Corollary 2.2. $\mathrm{PG}(\widehat{V})$ is projectively equivalent to $\mathrm{PG}(5, q)$ in $\mathrm{PG}\left(5, q^{2}\right)$.

We call the the projective space $\mathrm{PG}(\widehat{V})$ the cyclic representation of $\mathrm{PG}(5, q)$ over $\operatorname{GF}\left(q^{2}\right)$.

Recall that a $2 \times 2 q$-circulant (or Dickson) matrix over $\operatorname{GF}\left(q^{2}\right)$ is a matrix of the form

$$
D=\left(\begin{array}{ll}
d_{1} & d_{2} \\
d_{2}^{q} & d_{1}^{q}
\end{array}\right)
$$

with $d_{1}, d_{2} \in \operatorname{GF}\left(q^{2}\right)$.

Let $\mathcal{B}$ denote the basis $\left\{v_{1}^{(i)}, v_{2}^{(i)}: i=1,2,3\right\}$ of $\widehat{V}$.

Proposition 2.3. In the basis $\mathcal{B}$, the matrix associated to any endomorphism of $\widehat{V}$ is of the form

$$
\left(\begin{array}{lll}
D_{11} & D_{12} & D_{13} \\
D_{21} & D_{22} & D_{23} \\
D_{31} & D_{32} & D_{33}
\end{array}\right),
$$

where $D_{i j}$ is a $2 \times 2$-circulant matrix over $\operatorname{GF}\left(q^{2}\right)$.

Proof. It is easily seen that any matrix of type (2.2) is associated to an endomorphism of $\widehat{V}$.

Conversely, take an endomorphism $\tau$ of $V\left(6, q^{2}\right)$ and let $T=\left(t_{i j}\right), t_{i j} \in \operatorname{GF}\left(q^{2}\right)$, be the matrix of $\tau$ in the basis $\mathcal{B}$. For a generic array $\mathbf{x}=\left(x, x^{q}, y, y^{q}, z, z^{q}\right) \in \widehat{V}$,

$$
T \mathbf{x}^{t}=\left(\begin{array}{c}
\vdots \\
t_{k, 1} x+t_{k, 2} x^{q}+t_{k, 3} y+t_{k, 4} y^{q}+t_{k, 5} z+t_{k, 6} z^{q} \\
\vdots
\end{array}\right), \text { for } k=1, \ldots, 6 .
$$

If $y=z=0$, a necessary condition for $T \mathbf{x}^{t} \in \widehat{V}$ is

$$
\left(t_{k, 1} x+t_{k, 2} x^{q}\right)^{q}=t_{k+1,1} x+t_{k+1,2} x^{q},
$$

for $k=1,3,5$, that is,

$$
\left(t_{k, 2}^{q}-t_{k+1,1}\right) x+\left(t_{k, 1}^{q}-t_{k+1,2}\right) x^{q}=0,
$$

for $k=1,3,5$ and for all $x \in \operatorname{GF}\left(q^{2}\right)$. This shows that the polynomial in $x$ of degree $q$ on the left hand side of the last equation has at least $q^{2}$ roots. Therefore, it must be the zero polynomial. Hence $t_{k+1,1}=t_{k, 2}^{q}$ and $t_{k+1,2}=t_{k, 1}^{q}$, for $k=1,3,5$. To end the proof, it is enough to repeat the above argument for $x=z=0$ and then for $x=y=0$.

Next we exhibit quadratic forms on $V\left(6, q^{2}\right)$ which induce quadratic forms on $\widehat{V}$.

The vector space $V(2 n, q)$ has precisely two (nondegenerate) quadratic forms, and they differ by their Witt-index, that is the dimension of their maximal totally singular subspaces; 
see $[22,32]$. These dimensions are $n-1$ and $n$, and the quadratic form is elliptic or hyperbolic, respectively. In terms of the associated projective space $\operatorname{PG}(2 n-1, q)$, the elliptic (resp. hyperbolic) quadratic form defines an elliptic (resp. hyperbolic) quadric of $\mathrm{PG}(2 n-1, q)$.

Fix a basis $\{1, \epsilon\}$ for $\operatorname{GF}\left(q^{2}\right)$ over $\operatorname{GF}(q)$, and write $x=x_{0}+\epsilon x_{1}$, for $x \in \operatorname{GF}\left(q^{2}\right)$ with $x_{0}, x_{1} \in \operatorname{GF}(q)$. Here, $\epsilon$ is taken such that $\epsilon^{2}=\xi$ with $\xi$ a nonsquare in $\operatorname{GF}(q)$ for $q$ odd, and that $\epsilon^{2}+\epsilon=s$ with $s \in C_{1}$ and $s \neq 1$ for $q$ even, where $C_{1}$ stands for the set of elements in $\operatorname{GF}(q)$ with absolute trace 1. Furthermore, Tr denotes the trace map $x \in \mathrm{GF}\left(q^{2}\right) \rightarrow x+x^{q} \in \mathrm{GF}(q)$.

Proposition 2.4. Let $\alpha, \beta \in \mathrm{GF}\left(q^{2}\right)$ satisfy the following conditions:

$$
\left\{\begin{array}{l}
4 \alpha^{q+1}+\left(\beta^{q}-\beta\right)^{2} \text { is nonsquare in } \mathrm{GF}(q), \text { for } q \text { odd }, \\
\alpha^{q+1} /\left(\beta^{q}+\beta\right)^{2} \in C_{0} \text { with } \beta \in \mathrm{GF}\left(q^{2}\right) \backslash \mathrm{GF}(q), \text { for } q \text { even, }
\end{array}\right.
$$

where $C_{0}$ stands for the set of elements in $\mathrm{GF}(q)$, q even, with absolute trace 0 . Let $Q_{\alpha, \beta}$ be the quadratic form on $V\left(6, q^{2}\right)$ given by

$$
\begin{aligned}
& Q_{\alpha, \beta}\left(X_{1}, X_{2}, Y_{1}, Y_{2}, Z_{1}, Z_{2}\right)= \\
& \delta^{q} X_{1} Z_{2}+\delta X_{2} Z_{1}+\alpha \delta Y_{1}^{2}+\alpha^{q} \delta^{q} Y_{2}^{2}+\operatorname{Tr}(\delta \beta) Y_{1} Y_{2},
\end{aligned}
$$

with $\delta=\epsilon$ or $\delta=1$ according as $q$ is odd or even. then the restriction $\widehat{Q}_{\alpha, \beta}$ of $Q_{\alpha, \beta}$ on $\widehat{V}$ defines an elliptic quadratic form on $\widehat{V}$.

Proof. Two cases are treated separately according as $q$ is odd or even.

If $q$ is odd, let $b_{\alpha, \beta}$ denote the symmetric bilinear form on $V\left(6, q^{2}\right)$ associated to $Q_{\alpha, \beta}$. The matrix of $b_{\alpha, \beta}$ in the canonical basis is

$$
B_{\alpha, \beta}=\left(\begin{array}{ccc}
O_{2} & O_{2} & E \\
O_{2} & A_{\alpha, \beta} & O_{2} \\
\bar{E} & O_{2} & O_{2}
\end{array}\right)
$$

with

$$
E=\left(\begin{array}{cc}
0 & \epsilon^{q} \\
\epsilon & 0
\end{array}\right), \quad \bar{E}=\left(\begin{array}{cc}
0 & \epsilon \\
\epsilon^{q} & 0
\end{array}\right) \quad \text { and } \quad A_{\alpha, \beta}=\left(\begin{array}{cc}
2 \alpha \epsilon & \operatorname{Tr}(\epsilon \beta) \\
\operatorname{Tr}(\epsilon \beta) & 2 \alpha^{q} \epsilon^{q}
\end{array}\right)
$$

A straightforward computation shows that $B_{\alpha, \beta}$ induces a symmetric bilinear form on $\widehat{V}$. Let $\widehat{Q}_{\alpha, \beta}$ denote the resulting quadratic form on $\widehat{V}$.

Since det $A_{\alpha, \beta}=4 \alpha^{q+1}+\left(\beta^{q}-\beta\right)^{2}$ is nonsquare in $\operatorname{GF}(q)$, it follows that $Q_{\alpha, \beta}$ is nondegenerate. Hence $\widehat{Q}_{\alpha, \beta}$ is nondegenerate, as well. Let $H$ be the four-dimensional subspace $\left\{\left(x, x^{q}, 0,0, z, z^{q}\right): x, z \in G F\left(q^{2}\right)\right\}$ of $\widehat{V}$. Then the restriction of $\widehat{Q}_{\alpha, \beta}$ on $H$ is a hyperbolic quadratic form, as $L_{1}=\left\{\left(x, x^{q}, 0,0,0,0\right): x \in \operatorname{GF}\left(q^{2}\right)\right\}$ and $L_{2}=$ $\left\{\left(0,0,0,0, z, z^{q}\right): z \in G F\left(q^{2}\right)\right\}$ are totally isotropic subspaces with trivial intersection. The orthogonal space of $H$ with respect to $b_{\alpha, \beta}$ is $L=\left\{\left(0,0, y, y^{q}, 0,0\right): y \in \operatorname{GF}\left(q^{2}\right)\right\}$. By [22, Proposition 2.5.11], $\widehat{Q}_{\alpha, \beta}$ is elliptic if and only if the restriction of $\widehat{Q}_{\alpha, \beta}$ on $L$ is elliptic, that is,

$$
\operatorname{Tr}\left(\alpha \epsilon y^{2}+\epsilon \beta y^{q+1}\right)=0
$$


has no solution $y \in \operatorname{GF}\left(q^{2}\right)$ other than 0 .

Write $y=y_{0}+\epsilon y_{1}, \alpha=a_{0}+\epsilon a_{1}$ and $\beta=b_{0}+\epsilon b_{1}$ with $y_{0}, y_{1}, a_{0}, a_{1}, b_{0}, b_{1} \in \operatorname{GF}(q)$. As $\epsilon^{q}=-\epsilon$ and $\epsilon^{2}=\xi$, we have

$$
\begin{aligned}
y^{q} & =y_{0}-\epsilon y_{1} \\
y^{q+1} & =y_{0}^{2}-\xi y_{1}^{2} \\
y^{2} & =y_{0}^{2}+\xi y_{1}^{2}+2 \epsilon y_{0} y_{1} \\
y^{2 q} & =y_{0}^{2}+\xi y_{1}^{2}-2 \epsilon y_{0} y_{1} \\
\alpha \epsilon y^{2} & =\xi\left(2 a_{0} y_{0} y_{1}+a_{1}\left(y_{0}^{2}+\xi y_{1}^{2}\right)\right)+\epsilon\left(a_{0}\left(y_{0}^{2}+\xi y_{1}^{2}\right)+2 \xi a_{1} y_{0} y_{1}\right) \\
\alpha^{q} \epsilon^{q} y^{2 q} & =\xi\left(2 a_{0} y_{0} y_{1}+a_{1}\left(y_{0}^{2}+\xi y_{1}^{2}\right)\right)-\epsilon\left(a_{0}\left(y_{0}^{2}+\xi y_{1}^{2}\right)+2 \xi a_{1} y_{0} y_{1}\right),
\end{aligned}
$$

whence

$$
\operatorname{Tr}\left(\alpha \epsilon y^{2}\right)=2 \xi\left(2 a_{0} y_{0} y_{1}+a_{1}\left(y_{0}^{2}+\xi y_{1}^{2}\right)\right)
$$

Moreover,

$$
\operatorname{Tr}\left(\epsilon \beta y^{q+1}\right)=2 \xi b_{1}\left(y_{0}^{2}-\xi y_{1}^{2}\right)
$$

Then Equation (2.4) has a nontrivial solution $y \in \operatorname{GF}\left(q^{2}\right)$ if and only if $\left(y_{0}, y_{1}\right) \neq(0,0)$ with $y_{0}, y_{1} \in \mathrm{GF}(q)$ is a solution of

$$
\left(a_{1}+b_{1}\right) y_{0}^{2}+2 a_{0} y_{0} y_{1}+\xi\left(a_{1}-b_{1}\right) y_{1}^{2}=0 .
$$

By a straightforward computation, (2.5) occurs if and only if $4 \alpha^{q+1}+\left(\beta^{q}-\beta\right)^{2}=u^{2}$ for some $u \in \operatorname{GF}(q)$. But the latter equation contradicts our hypothesis. Therefore, Equation (2.4) has no nontrivial solution in $\operatorname{GF}\left(q^{2}\right)$ and hence $\widehat{Q}_{\alpha, \beta}$ is elliptic.

For $q$ even, the above approach still works up to some differences due to the fact that the well known formula solving equations of degree 2 fails in even characteristic. For completeness, we give all details.

If $q$ is even, the restriction of $Q_{\alpha, \beta}$ on $\widehat{V}$ is a quadratic form $\widehat{Q}_{\alpha, \beta}$ on $\widehat{V}$, and the matrix of the associated bilinear form $b_{\beta}$ is

$$
B_{\beta}=\left(\begin{array}{ccc}
O_{2} & O_{2} & E \\
O_{2} & A_{\beta} & O_{2} \\
E & O_{2} & O_{2}
\end{array}\right)
$$

where

$$
E=\left(\begin{array}{ll}
0 & 1 \\
1 & 0
\end{array}\right) \quad \text { and } \quad A_{\beta}=\left(\begin{array}{cc}
0 & \operatorname{Tr}(\beta) \\
\operatorname{Tr}(\beta) & 0
\end{array}\right)
$$

Since $\beta \notin \mathrm{GF}(q)$, a straightforward computation shows that the radical of $b_{\beta}$ is trivial, which gives $\widehat{Q}_{\alpha, \beta}$ is nonsingular. As for the odd $q$ case, the orthogonal space of $H$ with respect to $b_{\beta}$ is $L$. Therefore, $\widehat{Q}_{\alpha, \beta}$ is elliptic if and only if

$$
\operatorname{Tr}\left(\alpha y^{2}+\beta y^{q+1}\right)=0
$$

has no nontrivial solution $y \in \operatorname{GF}\left(q^{2}\right)$. 
As before, let $y=y_{0}+\epsilon y_{1}, \alpha=a_{0}+\epsilon a_{1}$ and $\beta=b_{0}+\epsilon b_{1}$ with $y_{0}, y_{1}, a_{0}, a_{1}, b_{0}, b_{1} \in$ $\operatorname{GF}(q)$. As $\epsilon^{q}=\epsilon+1$ and $\epsilon^{2}=\epsilon+s$, with $s \in C_{1}$, we have

$$
\begin{aligned}
y^{q} & =y_{0}+y_{1}+\epsilon y_{1} \\
y^{q+1} & =y_{0}^{2}+y_{0} y_{1}+s y_{1}^{2} \\
y^{2} & =y_{0}^{2}+s y_{1}^{2}+\epsilon y_{1}^{2} \\
y^{2 q} & =y_{0}^{2}+(s+1) y_{1}^{2}+\epsilon y_{1}^{2} \\
\alpha y^{2}= & a_{0} y_{0}^{2}+s\left(a_{0}+a_{1}\right) y_{1}^{2}+\epsilon\left(a_{0} y_{1}^{2}+a_{1} y_{0}^{2}+(s+1) a_{1} y_{1}^{2}\right) \\
\alpha^{q} y^{2 q}= & a_{0} y_{0}^{2}+s\left(a_{0}+a_{1}\right) y_{1}^{2}+\left(a_{0} y_{1}^{2}+a_{1} y_{0}^{2}+(s+1) a_{1} y_{1}^{2}\right) \\
& \quad+\epsilon\left(a_{0} y_{1}^{2}+a_{1} y_{0}^{2}+(s+1) a_{1} y_{1}^{2}\right),
\end{aligned}
$$

whence

$$
\operatorname{Tr}\left(\alpha y^{2}\right)=a_{0} y_{1}^{2}+a_{1} y_{0}^{2}+(s+1) a_{1} y_{1}^{2},
$$

and

$$
\operatorname{Tr}\left(\beta y^{q+1}\right)=b_{1}\left(y_{0}^{2}+y_{0} y_{1}+s y_{1}^{2}\right) .
$$

Therefore, Equation (2.6) has a nontrivial solution in $\operatorname{GF}\left(q^{2}\right)$ if and only if

$$
\left(a_{1}+b_{1}\right) y_{0}^{2}+b_{1} y_{0} y_{1}+\left(a_{0}+a_{1}+s a_{1}+s b_{1}\right) y_{1}^{2}=0 .
$$

Assume $y=y_{0} \in \mathrm{GF}(q)$ is a nontrivial solution of (2.6). Then $a_{1}=b_{1}$. This gives

$$
\frac{\alpha^{q+1}}{\left(\beta^{q}+\beta\right)^{2}}=\frac{a_{0}^{2}}{a_{1}^{2}}+\frac{a_{0}}{a_{1}}+s \in C_{1},
$$

a contradiction since

$$
\frac{a_{0}^{2}}{a_{1}^{2}}+\frac{a_{0}}{a_{1}} \in C_{0} .
$$

Assume that $y=y_{0}+\epsilon y_{1} \in \operatorname{GF}\left(q^{2}\right)$, with $y_{1} \neq 0$, is a solution of (2.6). Then $y_{0} y_{1}^{-1}$ is a solution of

$$
\left(a_{1}+b_{1}\right) X^{2}+b_{1} X+a_{0}+a_{1}+s\left(a_{1}+b_{1}\right)=0,
$$

where $b_{1} \neq 0$.

Let $Y=\left(a_{1}+b_{1}\right) b_{1}^{-1} X$. Replacing $X$ by $Y$ in (2.7) gives $Y^{2}+Y+d=0$ where

$$
d=\frac{a_{0}^{2}+a_{1} a_{0}+s a_{1}^{2}}{b_{0}^{2}}+\frac{a_{0}^{2}+a_{1}^{2}}{b_{0}^{2}}+\frac{a_{0}+a_{1}}{b_{0}}+s .
$$

Here, $d \in C_{1}$ by

$$
\frac{a_{0}^{2}+a_{1} a_{0}+s a_{1}^{2}}{b_{0}^{2}}=\frac{\alpha^{q+1}}{\left(\beta^{q}+\beta\right)^{2}} \in C_{0} .
$$

This shows that Equation (2.7) has no nontrivial solution in $\operatorname{GF}(q)$. Hence Equation (2.6) has no nontrivial solution in $\operatorname{GF}\left(q^{2}\right)$, as well. Therefore $\widehat{Q}_{\alpha, \beta}$ is elliptic.

Let $\widehat{\mathcal{Q}}_{\alpha, \beta}$ stand for the elliptic quadric in $\operatorname{PG}(\widehat{V})$ defined by the quadratic form $\widehat{Q}_{\alpha, \beta}$ on $\widehat{V}$. Then the coordinates of the points of $\operatorname{PG}(\widehat{V})$ that lie on $\widehat{\mathcal{Q}}_{\alpha, \beta}$ satisfy the equation

$$
\delta^{q} X Z^{q}+\delta X^{q} Z+\alpha \delta Y^{2}+\alpha^{q} \delta^{q} Y^{2 q}+\operatorname{Tr}(\delta \beta) Y^{q+1}=0,
$$

with $\delta=\epsilon$ or $\delta=1$ according as $q$ is odd or even. 


\section{The GF( $q$ )-linear representation of Buekenhout-Metz unitals}

In the light of Proposition 2.1, we introduce another incidence structure $\Pi(\widehat{\mathcal{S}})$.

Let $\widehat{\phi}$ be the bijective map defined by

$$
\begin{array}{cccc}
\widehat{\phi}: & V\left(3, q^{2}\right) & \longrightarrow & \widehat{V} \\
& (x, y, z) & \longmapsto & \left(x, x^{q}, y, y^{q}, z, z^{q}\right)
\end{array} .
$$

By Proposition 2.1, $\widehat{\phi}$ is the field reduction of $V\left(3, q^{2}\right)$ over $\operatorname{GF}(q)$ in the basis $\left\{v_{1}^{(i)}, v_{2}^{(i)}, i=1,2,3\right\}$ of $V\left(6, q^{2}\right)$.

The points of $\operatorname{PG}\left(2, q^{2}\right)$ are mapped by $\widehat{\phi}$ to the two-dimensional $\operatorname{GF}(q)$-subspaces of $\widehat{V}$ of the form

$$
\left\{\left(\lambda x, \lambda^{q} x^{q}, \lambda y, \lambda^{q} y^{q}, \lambda z, \lambda^{q} z^{q}\right): \lambda \in \mathrm{GF}\left(q^{2}\right)\right\}, \text { for } x, y, z \in \operatorname{GF}\left(q^{2}\right),
$$

and hence lines of $\mathrm{PG}(\widehat{V})$. Such lines form a line-spread $\widehat{\mathcal{S}}$ of $\mathrm{PG}(\widehat{V})$. By Proposition 2.1 and Corollary 2.2, $\widehat{\mathcal{S}}$ is projectively equivalent to $\mathcal{S}$ in $\mathrm{PG}\left(5, q^{2}\right)$. Hence, $\widehat{\mathcal{S}}$ is also a Desarguesian line-spread of $\mathrm{PG}(\widehat{V})$. Therefore, in $\mathrm{PG}\left(5, q^{2}\right) \Pi(\widehat{\mathcal{S}})$ is projectively equivalent to the $\mathrm{GF}(q)$-linear representation $\Pi(\mathcal{S})$ of $\mathrm{PG}\left(2, q^{2}\right)$.

The following lemma goes back to Singer, see [31].

Lemma 3.1. Let $\omega$ be a primitive element of $\operatorname{GF}\left(q^{2}\right)$ over $\operatorname{GF}(q)$ with minimal polynomial $f(T)=T^{2}-p_{1} T-p_{0}$. then the multiplication by $\omega$ in $\mathrm{GF}\left(q^{2}\right)$ defines a Singer cycle of $V(2, q)=\{(a, b): a, b \in \mathrm{GF}(q)\}$ whose matrix is the companion matrix of $f(T)$.

Proposition 3.2. Any endomorphism of $V\left(3, q^{2}\right)$ with matrix $A=\left(a_{i j}\right)$ defines the endomorphism of $\widehat{V}$ with matrix

$$
\left(\begin{array}{lll}
D_{11} & D_{12} & D_{13} \\
D_{21} & D_{22} & D_{23} \\
D_{31} & D_{32} & D_{33}
\end{array}\right)
$$

where $D_{i j}=\operatorname{diag}\left(a_{i j}, a_{i j}^{q}\right)$.

The Frobenius transformation $\psi:(x, y, z) \mapsto\left(x^{q}, y^{q}, z^{q}\right)$ of $V\left(3, q^{2}\right)$ defines the endomorphism of $\widehat{V}$ with matrix

$$
\left(\begin{array}{ccc}
\widehat{F} & 0 & 0 \\
0 & \widehat{F} & 0 \\
0 & 0 & \widehat{F}
\end{array}\right),
$$

where

$$
\widehat{F}=\left(\begin{array}{ll}
0 & 1 \\
1 & 0
\end{array}\right)
$$

Proof. The Singer cycle defined by a primitive element $\omega$ of $\operatorname{GF}\left(q^{2}\right)$ over $\operatorname{GF}(q)$ acts on the $\operatorname{GF}(q)$-vector space $\left\{\left(x, x^{q}\right): x \in \operatorname{GF}\left(q^{2}\right)\right\}$ by the matrix $D=\operatorname{diag}\left(\omega, \omega^{q}\right)$. For every entry $a_{i j}$ of $A$, write $a_{i j}=\omega^{e(i, j)}, 0 \leq e(i, j) \leq q^{2}-2$. From Lemma 3.1, the multiplication by $a_{i j}$ in $\operatorname{GF}\left(q^{2}\right)$ defines the endomorphism with matrix $D^{e(i, j)}=\operatorname{diag}\left(a_{i j}, a_{i j}^{q}\right)$. From this the first part of the proposition follows. The second part comes from Cooperstein's paper [14]. 
Remark 3.3. From a result due to Dye [16], the stabilizer of the Desarguesian partition $\mathcal{K}$ in $\operatorname{GL}(6, q)$ is the semidirect product of the field extension subgroup $\operatorname{GL}\left(3, q^{2}\right)$ by the cyclic subgroup $\langle\psi\rangle$ generated by the Frobenius transformation. In terms of projective geometry, the stabilizer of the Desarguesian spread $\mathcal{S}$ in $\operatorname{PGL}(6, q)$ is $\operatorname{GL}\left(3, q^{2}\right) \rtimes$ $\langle\psi\rangle) / \operatorname{GF}(q)^{*}[16]$. It should be noted that the center of $\operatorname{GL}(\widehat{V})$ is the subgroup $\left\{c I: c \in \operatorname{GF}(q)^{*}\right\}$. Proposition 3.2 provides the representation in $\operatorname{GL}(\widehat{V})$ and $\operatorname{PGL}(\widehat{V})$ of these stabilizers.

In [2] and [17] the orthogonal Buekenhout-Metz unitals are coordinatized in PG $\left(2, q^{2}\right)$. Let $L_{\infty}$ be the line of $\operatorname{PG}\left(2, q^{2}\right)$ with equation $Z=0$ and $P_{\infty}=\langle(1,0,0)\rangle_{q^{2}}$.

Theorem 3.4. Let $\alpha, \beta \in \mathrm{GF}\left(q^{2}\right)$ such that

$$
\left\{\begin{array}{l}
4 \alpha^{q+1}+\left(\beta^{q}-\beta\right)^{2} \text { is nonsquare in } \mathrm{GF}(q), \text { for } q \text { odd } \\
\alpha^{q+1} /\left(\beta^{q}+\beta\right)^{2} \in C_{0} \text { with } \beta \in \mathrm{GF}\left(q^{2}\right) \backslash \mathrm{GF}(q), \text { for } q \text { even. }
\end{array}\right.
$$

Then

$$
U_{\alpha, \beta}=\left\{\left\langle\left(\alpha y^{2}+\beta y^{q+1}+r, y, 1\right)\right\rangle_{q^{2}}: y \in \mathrm{GF}\left(q^{2}\right), r \in \mathrm{GF}(q)\right\} \cup\left\{P_{\infty}\right\}
$$

is an orthogonal Buekenhout-Metz unital. $U_{\alpha, \beta}$ is classical if and only if $\alpha=0$.

Conversely, every orthogonal Buekenhout-Metz unital can be expressed as $U_{\alpha, \beta}$ for some $\alpha, \beta \in \mathrm{GF}\left(q^{2}\right)$ which satisfy the above conditions.

We go back to the projective equivalence of $\Pi(\mathcal{S})$ and $\Pi(\widehat{\mathcal{S}})$ arising from the bijective map $\widehat{\phi}$. The line set $\widehat{\phi}\left(U_{\alpha, \beta}\right)=\left\{\widehat{\phi}(P): P \in U_{\alpha, \beta}\right\}$ can be regarded as the restriction on $U_{\alpha, \beta}$ of the $\mathrm{GF}(q)$-linear representation of $\operatorname{PG}\left(2, q^{2}\right)$ in $\operatorname{PG}(\widehat{V})$.

Remark 3.5. Thas [33] showed that the $\operatorname{GF}(q)$-linear representation of the classical unital is a partition of an elliptic quadric in $\operatorname{PG}(5, q)$. Thas's result is obtained here when the representation $\widehat{\phi}\left(U_{0, \beta}\right)$ is used. Let $\delta=\epsilon$ for odd $q$, and $\delta=1$ for even $q$. For any $\beta \in \operatorname{GF}\left(q^{2}\right)$ satisfying the conditions of Theorem 3.4, $U_{0, \beta}$ is the set of absolute points of the unitary polarity associated to the Hermitian form $h_{\beta}$ of $V\left(3, q^{2}\right)$ with matrix

$$
H_{\beta}=\left(\begin{array}{ccc}
0 & 0 & \delta^{q} \\
0 & \operatorname{Tr}(\delta \beta) & 0 \\
\delta & 0 & 0
\end{array}\right)
$$

Hence $U_{0, \beta}$ has equation

$$
\delta X^{q} Z+\delta^{q} X Z^{q}+\operatorname{Tr}(\delta \beta) Y^{q+1}=0 .
$$

Let $\operatorname{Tr}$ denote the trace map of $\operatorname{GF}\left(q^{2}\right)$ over $\operatorname{GF}(q)$. For any $v, v^{\prime} \in V\left(3, q^{2}\right)$,

$$
\operatorname{Tr}\left(h_{\beta}\left(v, v^{\prime}\right)\right)= \begin{cases}b_{0, \beta}\left(\widehat{\phi}(v), \widehat{\phi}\left(v^{\prime}\right)\right), & \text { for } q \text { odd } \\ b_{\beta}\left(\widehat{\phi}(v), \widehat{\phi}\left(v^{\prime}\right)\right), & \text { for } q \text { even }\end{cases}
$$

This shows that the points in $\widehat{\phi}\left(U_{0, \beta}\right)$ belong to $\widehat{\mathcal{Q}}_{0, \beta}$. In particular, the line set $\widehat{\phi}\left(U_{\alpha, \beta}\right)$ is a partition of $\widehat{\mathcal{Q}}_{0, \beta}$. 
We now put in evidence the relation between the elliptic quadric $\widehat{\mathcal{Q}}_{\alpha, \beta}$ and the Buekenhout representation of $U_{\alpha, \beta}$ in the Andrè/Bruck-Bose model of $\operatorname{PG}\left(2, q^{2}\right)$.

The subspace $\Lambda=\left\{\left\langle\left(x, x^{q}, y, y^{q}, c, c\right)\right\rangle_{q}: c \in \mathrm{GF}(q), x, y \in \mathrm{GF}\left(q^{2}\right)\right\}$ is an hyperplane of $\mathrm{PG}(\widehat{V})$ containing the 3-dimensional subspace $\Sigma=\left\{\left\langle\left(x, x^{q}, y, y^{q}, 0,0\right)\right\rangle_{q}\right.$ : $\left.x, y \in \operatorname{GF}\left(q^{2}\right)\right\}$. The line set $\mathcal{N}=\left\{\widehat{\phi}(P): P \in L_{\infty}\right\}$ is a Desarguesian line spread of $\Sigma$. Hence, $\mathcal{N}$ defines the Andrè/Bruck-Bose model of $\mathrm{PG}\left(2, q^{2}\right)$ in $\Lambda$ : the points are the lines of $\mathcal{N}$ and the points of $\Lambda$ not in $\Sigma$, the lines are the planes of $\Lambda$ not in $\Sigma$ which meet $\Sigma$ in a line of $\mathcal{N}$ and $\mathcal{N}$ itself, incidence is defined by inclusion. We denote by $\pi(\mathcal{N})$ this model of $\operatorname{PG}\left(2, q^{2}\right)$. The set $\bar{U}_{\alpha, \beta}=\bigcup_{P \in U_{\alpha, \beta}}(\widehat{\phi}(P) \cap \Lambda)$ is the Buekenhout representation of $U_{\alpha, \beta}$ in $\pi(\mathcal{N})$.

The hyperplane $\Lambda$ is the orthogonal space of the point $R=\langle(1,1,0,0,0,0)\rangle_{q}$ with respect the polarity associated with the quadric $\widehat{\mathcal{Q}}_{\alpha, \beta}$. Since $R \in \widehat{\mathcal{Q}}_{\alpha, \beta}$, the intersection between $\Lambda$ and $\widehat{\mathcal{Q}}_{\alpha, \beta}$ is a cone $\Gamma_{\alpha, \beta}$ projecting an elliptic quadric from $R$ and containing the spread element $\widehat{\phi}\left(P_{\infty}\right)=\left\{\left\langle\left(x, x^{q}, 0,0,0,0\right)\right\rangle_{q}: x \in \mathrm{GF}\left(q^{2}\right)\right\}$ as a generator.

Proposition 3.6. The cone $\Gamma_{\alpha, \beta}$ coincides with the Buekenhout representation $\bar{U}_{\alpha, \beta}$ of $U_{\alpha, \beta}$ in $\pi(\mathcal{N})$, that is,

$$
\bigcup_{P \in U_{\alpha, \beta}}(\widehat{\phi}(P) \cap \Lambda)=\Gamma_{\alpha, \beta} .
$$

Proof. We have $\widehat{\phi}\left(P_{\infty}\right)=\widehat{\mathcal{Q}}_{\alpha, \beta} \cap \Sigma$. For any $P=\left\langle\left(a y^{2}+\beta y^{q+1}, y, 1\right)\right\rangle_{q^{2}} \in U_{\alpha, \beta}$,

$$
\widehat{\phi}(P)=\left\{\left\langle\left(\lambda\left(a y^{2}+\beta y^{q+1}\right), \lambda^{q}\left(a^{q} y^{2^{q}}+\beta^{q} y^{q+1}\right), \lambda y, \lambda^{q} y^{q}, \lambda, \lambda^{q}\right)\right\rangle_{q}: \lambda \in \operatorname{GF}\left(q^{2}\right)\right\} .
$$

Then $\widehat{\phi}(P) \cap \Lambda=\left\langle\left(\alpha y^{2}+\beta y^{q+1}+r, \alpha^{q} y^{2 q}+\beta^{q} y^{q+1}+r, y, y^{q}, 1,1\right)\right\rangle_{q}$. From a straightforward calculation involving Equation (2.8) of $\widehat{\mathcal{Q}}_{\alpha, \beta}$ it follows that $\widehat{\phi}(P) \cap \Lambda \in \Gamma_{\alpha, \beta}$. Since the size of $\bigcup_{P \in U_{\alpha, \beta} \backslash\left\{P_{\infty}\right\}}(\widehat{\phi}(P) \cap \Lambda)$ equals the size of $\Gamma_{\alpha, \beta} \backslash \widehat{\phi}\left(P_{\infty}\right)$ the result follows.

Remark 3.7. The affine points of $\Gamma_{\alpha, \beta}$ satisfy the equation

$$
\delta^{q} X+\delta X^{q}+\alpha \delta Y^{2}+\alpha^{q} \delta^{q} Y^{2 q}+\operatorname{Tr}(\delta \beta) Y^{q+1}=0,
$$

with $\delta=\epsilon$ or $\delta=1$ according as $q$ is odd or even. It may be observed that Equation (3.1) is the equation of the affine points of $U_{\alpha, \beta}[13,20]$. Equation (3.1) in homogeneous form is

$$
\delta^{q} X Z^{2 q-1}+\delta X^{q} Z^{q}+\alpha \delta Y^{2} Z^{2 q-2}+\alpha^{q} \delta^{q} Y^{2 q}+\operatorname{Tr}(\delta \beta) Y^{q+1} Z^{q-1}=0,
$$

which is satisfied by the points of the $\operatorname{GF}(q)$-linear representation $\widehat{\phi}\left(U_{\alpha, \beta}\right)$ of $U_{\alpha, \beta}$.

In [28], Polverino proved that the $\mathrm{GF}(q)$-linear representation of an orthogonal Buekenhout-Metz unital cover the $\operatorname{GF}(q)$-points of an algebraic hypersurface of degree four minus the complements of a line in a three-dimensional subspace. She also showed that the hypersurface is reducible if and only if the unital is classical. Polverino's result is obtained here when the representation $\widehat{\phi}\left(U_{0, \beta}\right)$ is used. Let $\mathcal{F}$ be the hypersurface of $\operatorname{PG}\left(5, q^{2}\right)$ with equation

$$
\mathcal{F}: \delta^{q} X_{1} Z_{1} Z_{2}^{2}+\delta X_{2} Z_{1}^{2} Z_{2}+\alpha \delta Y_{1}^{2} Z_{2}^{2}+\alpha^{q} \delta^{q} Y_{2}^{2} Z_{1}^{2}+\operatorname{Tr}\left(\delta \beta^{q}\right) Y_{1} Y_{2} Z_{1} Z_{2}=0 .
$$


The intersection $\widehat{\mathcal{F}}$ of $\mathcal{F}$ with $\mathrm{PG}(\widehat{V})$ consists of all points of $\mathrm{PG}(\widehat{V})$ satisfying the equation

$$
\delta^{q} X Z^{2 q+1}+\delta X^{q} Z^{q+2}+\alpha \delta Y^{2} Z^{2 q}+\alpha^{q} \delta^{q} Y^{2 q} Z^{2}+\operatorname{Tr}\left(\delta \beta^{q}\right) Y^{q+1} Z^{q+1}=0 .
$$

Clearly, $\widehat{\mathcal{F}}$ contains the three-dimensional subspace $\Sigma$. By the above arguments, the $\operatorname{GF}(q)$ linear representation $\widehat{\phi}\left(U_{\alpha, \beta}\right)$ covers the points in $\widehat{\mathcal{F}}$ minus the complements of $\widehat{\phi}\left(L_{\infty}\right)$ in $\Sigma$. Furthermore, Equation (3.2) defines an algebraic hypersurface of degree four of $\mathrm{PG}(5, q)$. A straightforward, though tedious, calculation shows that Equation (3.2) is precisely the algebraic hypersurface provided by Polverino in [28].

As elliptic quadrics in $\mathrm{PG}(\widehat{V})$ are projectively equivalent, some linear collineation $\tau_{\alpha}$ of PG( $(\widehat{V})$ takes $\widehat{\mathcal{Q}}_{0, \beta}$ to $\widehat{\mathcal{Q}}_{\alpha, \beta}$. Actually we need such a linear collineation $\tau_{\alpha}$ with some extra-property.

Proposition 3.8. In $\mathrm{PG}(\widehat{V})$ there exists a linear collineation $\tau_{\alpha}$ which takes $\widehat{\mathcal{Q}}_{0, \beta}$ to $\widehat{\mathcal{Q}}_{\alpha, \beta}$, preserves the subspaces $\Lambda, \Sigma$, and fixes $\widehat{\phi}\left(P_{\infty}\right)$ pointwise. Therefore it maps the cone $\Gamma_{0, \beta}$ into $\Gamma_{\alpha, \beta}$.

Proof. The restriction $\left.\widehat{Q}_{\alpha, \beta}\right|_{L}$ on the subspace $L=\left\{\left(0,0, y, y^{q}, 0,0\right): y \in \operatorname{GF}\left(q^{2}\right)\right\}$ of $\widehat{Q}_{\alpha, \beta}$ given by (2.3) is the quadratic form defined by

$$
\left.\widehat{Q}_{\alpha, \beta}\right|_{L}\left(y, y^{q}\right)=\alpha \delta y^{2}+\alpha^{q} \delta^{q} y^{2 q}+\operatorname{Tr}(\delta \beta) y^{q+1} \in \mathrm{GF}(q)
$$

which is of elliptic type by the proof of Proposition 2.4. As two such forms are equivalent, some endomorphism of $L$ maps $\left.\widehat{Q}_{0, \beta}\right|_{L}$ to $\left.\widehat{Q}_{\alpha, \beta}\right|_{L}$. In a natural way, as in the proof of Proposition 2.3, we may identify any endomorphism of $L$ with a $2 \times 2 q$-circulant matrix. Doing so, the endomorphism with matrix

$$
D=\left(\begin{array}{ll}
d_{1} & d_{2} \\
d_{2}^{q} & d_{1}^{q}
\end{array}\right),
$$

where

$$
\begin{aligned}
d_{1}^{q+1}+d_{2}^{q+1} & =1 \\
d_{1} d_{2}^{q} & =\alpha \delta \operatorname{Tr}(\delta \beta)^{-1},
\end{aligned}
$$

maps $\left.\widehat{Q}_{0, \beta}\right|_{L}$ to $\left.\widehat{Q}_{\alpha, \beta}\right|_{L}$. Let $\tau_{\alpha}$ be the linear collineation of $\mathrm{PG}(\widehat{V})$ defined by the matrix

$$
D_{\alpha}=\left(\begin{array}{ccc}
I_{2} & O_{2} & O_{2} \\
O_{2} & D & O_{2} \\
O_{2} & O_{2} & I_{2}
\end{array}\right)
$$

It is easily seen that $\tau_{\alpha}$ preserves the subspaces $\Lambda, \Sigma$, and fixes $\widehat{\phi}\left(P_{\infty}\right)$ pointwise, and that it maps the cone $\Gamma_{0, \beta}$ into $\Gamma_{\alpha, \beta}$.

Remark 3.9. Bearing in mind Remark 3.3, one can ask whether $\tau_{\alpha}$ is an incidence preserving map of $\Pi(\widehat{\mathcal{S}})$. The answer is negative by $d_{1} d_{2} \neq 0$ and Proposition 3.2. This implies that $\Gamma_{0, \beta}$ and $\Gamma_{\alpha, \beta}$ are Buekenhout representations of unitals of $\operatorname{PG}\left(2, q^{2}\right)$ and that they are not projectively equivalent. In particular, this provides a new proof for the existence of non-classical unitals embedded in $\mathrm{PG}\left(2, q^{2}\right)$. 
It is clear that the image $\widehat{\mathcal{S}}^{\tau_{\alpha}}$ of the Desarguesian line-spread $\widehat{\mathcal{S}}$ under the linear collineation $\tau_{\alpha}$ is a Desarguesian line-spread and it defines the $\operatorname{GF}(q)$-linear representation $\Pi\left(\widehat{\mathcal{S}}^{\tau_{\alpha}}\right)$ of $\operatorname{PG}\left(2, q^{2}\right)$.

\section{The proof of the Main Theorem}

In our proof the models of $\mathrm{PG}\left(2, q^{2}\right)$ treated in Section 3 play a role. Two of them arose from Desarguesian line-spreads of $\mathrm{PG}(\widehat{V})$ denoted by $\widehat{\mathcal{S}}$ and $\widehat{\mathcal{S}}^{\tau_{\alpha}}$ respectively, the third was the Andrè/Bruck-Bose model $\pi(\mathcal{N})$ in the 4-dimensional subspace $\Lambda$.

In $\mathrm{PG}\left(2, q^{2}\right)$ consider a unital $\mathcal{U}$ isomorphic, as a block-design, to an orthogonal Buekenhout-Metz unital $U_{\alpha, \beta}$ with $\alpha \neq 0$. It is known [2,17] that $U_{\alpha, \beta}$ has a special point which is the unique fixed point of the automorphism group of $U_{\alpha, \beta}$. Hence the automorphism group of $\mathcal{U}$ fixes a unique point of $\mathcal{U}$. Up to a change of the homogeneous coordinate system in $\mathrm{PG}\left(2, q^{2}\right)$, the special point of $U_{\alpha, \beta}$ is $P_{\infty}=\langle(1,0,0)\rangle_{q^{2}}$ and the tangent line of $U_{\alpha, \beta}$ at $P_{\infty}$ is $L_{\infty}: Z=0$. Up to a linear collineation, $P_{\infty} \in \mathcal{U}$ is the fixed point of the automorphism group of $\mathcal{U}$ and $L_{\infty}$ is the tangent to $\mathcal{U}$ at $P_{\infty}$. Therefore, $\mathcal{U}$ and $U_{\alpha, \beta}$ share $P_{\infty}$ and $L_{\infty}$.

We interpret the isomorphism between $\mathcal{U}$ and $U_{\alpha, \beta}$ in each of the above three models of $\mathrm{PG}\left(2, q^{2}\right)$. The representation $\widehat{\mathcal{U}}=\{\widehat{\phi}(P): P \in \mathcal{U}\}$ of $\mathcal{U}$ in $\Pi(\widehat{\mathcal{S}})$ is isomorphic, as a block-design, to $\widehat{U}_{\alpha, \beta}=\left\{\widehat{\phi}(P): P \in U_{\alpha, \beta}\right\}$. The Buekenhout representation $\overline{\mathcal{U}}=\bigcup_{P \in \mathcal{U}}(\widehat{\phi}(P) \cap \Lambda)$ of $\mathcal{U}$ in $\pi(\mathcal{N})$ is isomorphic, as a block-design, to $\bar{U}_{\alpha, \beta}=\bigcup_{P \in U_{\alpha, \beta}}(\widehat{\phi}(P) \cap \Lambda)$. Here, by Proposition 3.6, $\bar{U}_{\alpha, \beta}$ is the cone $\Gamma_{\alpha, \beta}$. This gives that the representation $\widetilde{\mathcal{U}}=\left\{L \in \widehat{\mathcal{S}}^{\tau_{\alpha}}: L \cap \Lambda \subset \bar{U}\right\}$ of $\mathcal{U}$ in $\Pi\left(\widehat{\mathcal{S}}^{\tau_{\alpha}}\right)$ is isomorphic, as a block-design, to $\widetilde{U}_{\alpha, \beta}=\left\{L \in \widehat{\mathcal{S}}^{\tau_{\alpha}}: L \cap \Lambda \subset \Gamma_{\alpha, \beta}\right\}$.

From Proposition 3.8, the lines which are the points of $\widetilde{U}_{\alpha, \beta}$ partition the elliptic quadric $\widehat{\mathcal{Q}}_{\alpha, \beta}=\widehat{\mathcal{Q}}_{0, \beta}^{\tau_{\alpha}}$. On the other hand, from Remark $3.5, \widehat{\mathcal{Q}}_{0, \beta}$ is partitioned by lines which are the points of the classical unital $\widehat{U}_{0, \beta}$ in $\Pi(\widehat{\mathcal{S}})$. This yields that $\widetilde{U}_{\alpha, \beta}$ coincides with $\widehat{U}_{0, \beta}^{\tau_{\alpha}}$. It turns out that $\widetilde{U}_{\alpha, \beta}$ is a classical unital in $\Pi\left(\widehat{\mathcal{S}}^{\tau_{\alpha}}\right)$, and hence $\widetilde{\mathcal{U}}$ is isomorphic, as a block-design, to the classical unital.

Now we quote the following result from [23] which was the keystone in the proof of Theorem 1.1.

Lemma 4.1. Let $\mathcal{U}$ be a unital embedded in a Desarguesian finite projective plane $\pi$ and isomorphic, as a block-design, to the classical unital. For any block $B$ of $\mathcal{U}$, let $\ell$ be the line of $\pi$ containing $B$. Then $B$ is an orbit of a cyclic subgroup of order $q+1$ contained in the projectivity group of $\ell$. This implies that $B$ is a Baer subline of $\ell$.

We emphasize that the proof of Lemma 4.1 only uses arguments involving point-block incidences of $\mathcal{U}$ viewed as a block-design embedded in $\pi$.

Therefore, Lemma 4.1 applies to $\widetilde{\mathcal{U}}$. Thus, every block of $\widetilde{\mathcal{U}}$ is a Baer subline of $\Pi\left(\widehat{\mathcal{S}}^{\tau_{\alpha}}\right)$, that is, a regulus of $\operatorname{PG}(\widehat{V})$. From this, each block of $\overline{\mathcal{U}}$ is the intersection of these reguli with $\Lambda$. In particular, each block of $\overline{\mathcal{U}}$ through $\widehat{\phi}\left(P_{\infty}\right)$ is the union of $\widehat{\phi}\left(P_{\infty}\right)$ with $q$ collinear affine points, and this implies that each block of $\widehat{\mathcal{U}}$ through $\widehat{\phi}\left(P_{\infty}\right)$ is a regulus of $\operatorname{PG}(\widehat{V})$ whose lines are in $\widehat{\mathcal{S}}$. Under $\widehat{\phi}$, these reguli correspond to Baer sublines of $\mathrm{PG}\left(2, q^{2}\right)$ through $P_{\infty}$. This yields that the points of $\mathcal{U}$ on each of the $q^{2}$ secant lines to $\mathcal{U}$ form a Baer subline through $P_{\infty}$. By the characterization of such unitals of $\operatorname{PG}\left(2, q^{2}\right)$ 
given in $[12,29]$, we may conclude that $\mathcal{U}$ is a Buekenhout-Metz unital. By definition, the Buekenhout representation $\overline{\mathcal{U}}$ of $\mathcal{U}$ is a cone that project an ovoid $\mathcal{O}$ from a point of $\widehat{\phi}\left(P_{\infty}\right)$ not in $\mathcal{O}$. Here an ovoid is a set of $q^{2}+1$ points in a 3-dimensional subspace of $\Lambda$ no three of which are collinear.

To conclude the proof we only need to prove that $\mathcal{O}$ is an elliptic quadric. Since the ovoids in $\mathrm{PG}(3, q)$ with odd $q$ are elliptic quadrics, see [4, 26], we assume $q=2^{h}$. In $\mathrm{PG}\left(3,2^{h}\right)$, there are known two ovoids, up to projectivities, namely the elliptic quadric which exist for $h \geq 1$, and the Tits ovoid which exists for odd $h \geq 3$; see [18, Chapter 10]. Let $\Omega$ be the 3-dimensional subspace of $\Lambda$ containing $\mathcal{O}$. Note that $\mathcal{O}=\Omega \cap \overline{\mathcal{U}}$. Set $\alpha_{\infty}$ to be the plane $\Omega \cap \Sigma$. Then $\alpha_{\infty}$ meets $\mathcal{O}$ exactly in the point $\mathcal{O} \cap \widehat{\phi}\left(P_{\infty}\right)$, and it is a simple matter to show that $\alpha_{\infty}$ contains only one line $\widehat{\phi}(P)$ of $\mathcal{N}$. Also, $\widehat{\phi}(P)$ is distinct from $\widehat{\phi}\left(P_{\infty}\right)$. Let $\alpha_{1}, \ldots, \alpha_{q}$ denote the further planes of $\Omega$ through $\widehat{\phi}(P)$. As these planes are lines of $\pi(\mathcal{N})$ through the point $\widehat{\phi}(P)$, each of them meets $\overline{\mathcal{U}}$ in 1 or $q+1$ points. This holds true for $\mathcal{O}$.

It is well known [19, Section 12.3] that in a finite Desarguesian projective plane through any point off a unital there are exactly $q+1$ tangent lines, that is, lines of the plane that intersects the unital in exactly one point. In terms of the unital $\overline{\mathcal{U}}$ this property states that there is only one plane among $\alpha_{1}, \ldots, \alpha_{q}$ that meets $\mathcal{O}$ in exactly one point. Let $\alpha_{1}$ denote this plane. Then the block $\alpha_{i} \cap \mathcal{O}$ of $\overline{\mathcal{U}}$, for $i=2, \ldots, q$, is the intersection of $\alpha_{i}$ with a regulus in $\operatorname{PG}(\widehat{V})$. Since that regulus does not contain $\widehat{\phi}(P)$, the block $\alpha_{i} \cap \mathcal{O}$ is a conic $C_{i}$ of $\alpha_{i}$, for $i=2, \ldots, q$. Thus the blocks $\alpha_{i} \cap \mathcal{O}$, for $i=2, \ldots, q$, are $q-1$ conics that partition all but two points of $\mathcal{O}$. By [8, Theorem 5] $\mathcal{O}$ is an elliptic quadric.

\section{References}

[1] J. André, Über nicht-Desarguessche Ebenen mit transitiver Translationsgruppe, Math. Z. 60 (1954), 156-186, doi:10.1007/bf01187370.

[2] R. D. Baker and G. L. Ebert, On Buekenhout-Metz unitals of odd order, J. Comb. Theory Ser. A 60 (1992), 67-84, doi:10.1016/0097-3165(92)90038-v.

[3] J. Bamberg, A. Betten, C. E. Praeger and A. Wassermann, Unitals in the Desarguesian projective plane of order 16, J. Statist. Plann. Inference 144 (2014), 110-122, doi:10.1016/j.jspi. 2012.10.006.

[4] A. Barlotti, Un'estensione del teorema di Segre-Kustaanheimo, Boll. Un. Mat. Ital. Serie 310 (1955), 498-506, http://www.bdim.eu/item?id=BUMI_1955_3_10_4_498_0.

[5] S. Barwick and G. Ebert, Unitals in Projective Planes, Springer Monographs in Mathematics, Springer, New York, 2008, doi:10.1007/978-0-387-76366-8.

[6] A. Betten, D. Betten and V. D. Tonchev, Unitals and codes, Discrete Math. 267 (2003), 23-33, doi:10.1016/s0012-365x(02)00600-3.

[7] R. C. Bose, On a representation of the Baer subplanes of the Desarguesian plane $\mathrm{PG}\left(2, q^{2}\right)$ in a projective five dimensional space $\mathrm{PG}(5, q)$, in: Colloquio Internazionale sulle Teorie Combinatorie, Tomo I, Accademia Nazionale dei Lincei, Rome, 1976 pp. 381-391, proceedings of a conference held in Rome, September 3 - 15, 1973.

[8] M. R. Brown, C. M. O'Keefe and T. Penttila, Triads, flocks of conics and $Q^{-}(5, q)$, Des. Codes Cryptogr. 18 (1999), 63-70, doi:10.1023/a:1008376900914.

[9] R. H. Bruck and R. C. Bose, The construction of translation planes from projective spaces, $J$. Algebra 1 (1964), 85-102, doi:10.1016/0021-8693(64)90010-9. 
[10] R. H. Bruck and R. C. Bose, Linear representations of projective planes in projective spaces, $J$. Algebra 4 (1966), 117-172, doi:10.1016/0021-8693(66)90054-8.

[11] F. Buekenhout, Existence of unitals in finite translation planes of order $q^{2}$ with a kernel of order q, Geom. Dedicata 5 (1976), 189-194, doi:10.1007/bf00145956.

[12] L. R. A. Casse, C. M. O'Keefe and T. Penttila, Characterizations of Buekenhout-Metz unitals, Geom. Dedicata 59 (1996), 29-42, doi:10.1007/bf00181524.

[13] D. B. Chandler, The sizes of the intersections of two unitals in $\mathrm{PG}\left(2, q^{2}\right)$, Finite Fields Appl. 25 (2014), 255-269, doi:10.1016/j.ffa.2013.10.001.

[14] B. N. Cooperstein, External flats to varieties in $\mathbb{P} G\left(M_{n, n}(\mathrm{GF}(q))\right)$, Linear Algebra Appl. 267 (1997), 175-186, doi:10.1016/s0024-3795(97)80049-3.

[15] N. Durante and A. Siciliano, Non-linear maximum rank distance codes in the cyclic model for the field reduction of finite geometries, Electron. J. Combin. 24 (2017), P2.33, https: / / www. combinatorics.org/ojs/index.php/eljc/article/view/v24i2p33.

[16] R. H. Dye, Spreads and classes of maximal subgroups of $\mathrm{GL}_{n}(q), \mathrm{SL}_{n}(q), \mathrm{PGL}_{n}(q)$ and $\mathrm{PSL}_{n}(q)$, Ann. Mat. Pura Appl. 158 (1991), 33-50, doi:10.1007/bf01759298.

[17] G. L. Ebert, On Buekenhout-Metz unitals of even order, European J. Combin. 13 (1992), 109117, doi:10.1016/0195-6698(92)90042-x.

[18] J. W. P. Hirschfeld, Finite Projective Spaces of Three Dimensions, Oxford Mathematical Monographs, Oxford University Press, New York, 1985.

[19] J. W. P. Hirschfeld, Projective Geometries over Finite Fields, Oxford Mathematical Monographs, Oxford University Press, New York, 2nd edition, 1998.

[20] J. W. P. Hirschfeld and G. Korchmáros, Arcs and curves over a finite field, Finite Fields Appl. 5 (1999), 393-408, doi:10.1006/ffta.1999.0260.

[21] A. M. W. Hui and P. P. W. Wong, On embedding a unitary block design as a polar unital and an intrinsic characterization of the classical unital, J. Comb. Theory Ser. A 122 (2014), 39-52, doi:10.1016/j.jcta.2013.09.007.

[22] P. Kleidman and M. Liebeck, The Subgroup Structure of the Finite Classical Groups, volume 129 of London Mathematical Society Lecture Note Series, Cambridge University Press, Cambridge, 1990, doi:10.1017/cbo9780511629235.

[23] G. Korchmáros, A. Siciliano and T. Szônyi, Embedding of classical polar unitals in PG $\left(2, q^{2}\right)$, J. Comb. Theory Ser. A 153 (2018), 67-75, doi:10.1016/j.jcta.2017.08.002.

[24] R. Metz, On a class of unitals, Geom. Dedicata 8 (1979), 125-126, doi:10.1007/bf00147935.

[25] G. P. Nagy and D. Mezőfi, UnitalSZ - a GAP package, Version 0.5, 23 March 2018, https : //nagygp.github.io/Unitalsz/.

[26] G. Panella, Caratterizzazione delle quadriche di uno spazio (tridimensionale) lineare sopra un corpo finito, Boll. Un. Mat. Ital. Serie 310 (1955), 507-513, http: / / www . bdim. eu/ item?id=BUMI_1955_3_10_4_507_0.

[27] T. Penttila and G. F. Royle, Sets of type $(m, n)$ in the affine and projective planes of order nine, Des. Codes Cryptogr. 6 (1995), 229-245, doi:10.1007/bf01388477.

[28] O. Polverino, Linear representation of Buekenhout-Metz unitals, Discrete Math. 267 (2003), 247-252, doi:10.1016/s0012-365x(02)00618-0.

[29] C. T. Quinn and R. Casse, Concerning a characterisation of Buekenhout-Metz unitals, J. Geom. 52 (1995), 159-167, doi:10.1007/bf01406836.

[30] B. Segre, Teoria di Galois, fibrazioni proiettive e geometrie non desarguesiane, Ann. Mat. Pura Appl. 64 (1964), 1-76, doi:10.1007/bf02410047. 
[31] J. Singer, A theorem in finite projective geometry and some applications to number theory, Trans. Amer. Math. Soc. 43 (1938), 377-385, doi:10.2307/1990067.

[32] D. E. Taylor, The Geometry of the Classical Groups, volume 9 of Sigma Series in Pure Mathematics, Heldermann Verlag, Berlin, 1992.

[33] J. A. Thas, Semipartial geometries and spreads of classical polar spaces, J. Comb. Theory Ser. A 35 (1983), 58-66, doi:10.1016/0097-3165(83)90026-2. 